\title{
Remotely detected high-field MRI of porous samples
}

\author{
Juliette A. Seeley, Song-I Han and Alexander Pines \\ Materials Sciences Division, Lawrence Berkeley National Laboratory, \\ Berkeley, California 94720, USA \\ AND \\ Department of Chemistry, University of California, \\ Berkeley, California 94720, USA
}

* Correspondence and requests for materials should be addressed to Alexander Pines

Prof. Dr. Alexander Pines

Chemistry Department, Hildebrand Hall

University of California at Berkeley

Berkeley, CA 94720, USA,

Fax: (510) 486-5744

Phone: (510) 642-1220

E-mail: pines@cchem.berkeley.edu 


\section{ABSTRACT}

Remote detection of NMR is a novel technique in which an NMR-active sensor surveys an environment of interest and retains memory of that environment to be recovered at a later time in a different location. The NMR or MRI information about the sensor nucleus is encoded and stored as spin polarization at the first location and subsequently moved to a different physical location for optimized detection. A dedicated probe incorporating two separate radio frequency (RF) circuits was built for this purpose. The encoding solenoid coil was large enough to fit around the bulky sample matrix, while the smaller detection solenoid coil had not only a higher quality factor but also an enhanced filling factor since the coil volume comprised purely the sensor nuclei. We obtained two-dimensional (2D) void space images of two model porous samples with resolution less than $1.4 \mathrm{~mm}^{2}$. The remotely-reconstructed images demonstrate the ability to determine fine structure with image quality superior to their directly detected counterparts and show the great potential of NMR remote detection for imaging applications that suffer from low sensitivity due to low concentrations and filling factor.

Keywords: Remote-detection, MRI, porous samples, laser-polarized xenon-129, sensitivity 


\section{INTRODUCTION}

The key feature of remote detection [1] is the spatial separation of the NMR encoding and detection steps, which allows for their separate optimization by providing the most suitable conditions for encoding without compromising detection quality. Parameters that influence the quality of NMR data such as static magnetic field strength, RF coil size and geometry, and even the type of detector can be chosen with a flexibility that conventional NMR would not permit (fig. 1). Remote detection is realized by employing a "signal carrier" to encode NMR information indicative of its environment. This time-domain or $k$-space information is stored point-by-point as spin polarization in the encoding location, each point being subsequently transported to the detection location. Remote detection is based on the principle of indirect detection of NMR (or phase encoding of MRI), which involves incrementing the time duration or $k$ value for which a transverse signal evolves. The resulting phase acquired after each evolution provides one point in the indirect signal. This naturally adds an extra dimension to the experiment. In a remote experiment, the indirect dimension provides information about the encoding environment, while the direct dimension simply gives the signal of the sensor in the detection region.

Sensitive modes of detection, that are challenging or not applicable in a direct experiment, such as SQUID [2] and optical detection [3] (fig. 1) and the sensitive detection achieved by narrowing the detection bandwidth (defined by $1 / T_{2}^{*}$ in conjunction with the appropriate filter) along the direct dimension [4-6], potentially offer a large enhancement for NMR and MRI. 
This technique adapts particularly well to laser-polarized Xe-129 NMR, not only because the highly polarized and chemically inert noble gas atom with long $T_{1}$ relaxation time is tailored for the role of an effective transport medium for spin polarization, but also because it provides a wealth of NMR-based physiochemical and biomedical information [7-13]. Thus, Xe-129 is a powerful sensor of its local environment and can retain information about that environment for a sufficient amount of time during transport. This Xe-129 guest, diluted inside the sample matrix, is extracted out of its host and subsequently transported and concentrated into the detector volume for more sensitive detection.

Xe-129 has been popular for NMR studies of various materials for two decades [9]. As a noble gas, xenon is non-reactive, making it a viable objective observer, yet with 54 electrons its chemical shift is extremely sensitive to its environment, even in the absence of covalent bonding. An additional advantage of xenon is its ability to be hyperpolarized through spin-exchange interaction with optically pumped $\mathrm{Rb}$ vapor [14-16]. A sensitivity enhancement of 5-6 orders of magnitude compared to thermal polarization can be realized.

NMR studies of materials such as porous media [17], solution [10, 18-20], and tissue (including in-vivo applications [11,12] and the xenon biosensor) by means of inclusion of sensor nuclei into the matrix inherently suffer from low signal-to-noise because the majority of the volume in the detection region is occupied by the matrix material itself. The guest sensor occupies only a small fraction of the total sample volume, resulting in a low coil filling factor or low magnetization, and thus decreased sensitivity. The sensitivity with which the 
sensor nuclei can be detected not only limits the signal-to-noise of the image but also its resolution, since the latter is determined by the minimum voxel size one can subdivide the total volume into by imposing strong gradient fields, such that the number of spins in one volume element remains detectable. Although laserpolarization of Xe-129 increases the signal-to-noise substantially, it does not change the insensitivity of the detection coil which has a low filling factor or the problem of low concentration of the relevant species.

Remote detection is a new technique [1] that addresses, among others, the problem of low sensitivity of NMR and MRI for material studies. Since the dilute "signal carrier" is separated out of the material's volume into the detector volume, more favorable conditions for detection can be chosen. Though our signal carrier is Xe-129, it is important to note that it is not the Xe-129 sample itself that is of interest, but its interactions with the encoding environment. Also note that other signal carrier fluids such as water or oil may be employed for remote detection MRI with the $T_{1}$ of these carriers being the only foreseeable limitation. There are numerous applications where the transportation of the fluids through a porous material is a natural part of the experiment [21]. Remote detection can easily be applied to such imaging and flow studies.

We will present an extension to our previous work in which remote detection is applied to imaging of porous samples by incorporating Xe-129 into the void space of materials. An enhancement of the void-space image is achieved through remote detection by using a separate coil with optimum filling factor for detection, different than that of the encoding coil containing the sample 
matrix. The expected sensitivity gain depends on many factors as discussed in Hoult's paper [22]. A simplified equation (eqn. 1) describing sensitivity may be used to calculate an order of magnitude approximation of sensitivity. The root mean squared signal-to-noise ratio $S$ is given by

$$
\begin{aligned}
S & =K \cdot \eta \cdot M_{0} \cdot V_{c} \sqrt{Q \cdot \omega_{0} \cdot n /\left(T \cdot \Delta f \cdot V_{c}\right)} \\
& =K \cdot m_{0} \cdot \sqrt{Q \cdot \omega_{0} \cdot n /\left(T \cdot \Delta f \cdot V_{c}\right)}
\end{aligned}
$$

where $K$ is a numerical factor which depends on coil geometry, the noise figure of the preamplifier, and also takes into account various physical constants. $\eta$ is the filling factor, $M_{0}$ is the nuclear magnetization, which takes into account the concentration of relevant spins in the sample volume, $V_{c}$ is the volume of the coil, $Q$ is the quality factor of the circuit, $\omega_{0}$ is the Larmor frequency, $n$ is the number of scans for signal accumulation, $T$ is the temperature of the probe, and $\Delta f$ is the detection bandwidth. $m_{0}$ in the second line of eqn. 1 is the net magnetic moment of the spins inside the coil volume, which is transported without loss from the encoding to the detection location in an ideal remote experiment. The signal-tonoise ratio in a remote experiment can be enhanced by optimizing the factors $\eta$, $M_{0}$ (via concentration), $V_{c}, Q, \omega_{0}, T$ and $\Delta f$ independently for the encoding and detection steps, which is not possible in a conventional NMR experiment. In remote detection NMR experiments, an RF coil with better $\eta$ and $Q$ can be used for detection than may be possible for the encoding coil, due to constraints imposed by the presence of the sample. A higher magnetic field can be employed for more sensitive detection while a lower field may be used for 
encoding, for example to decrease susceptibility artifacts in imaging. The detection coil can be cooled to enhance detection sensitivity while still preserving the optimal temperature conditions for the sample. The detection bandwidth may be narrowed by the application of pulse sequences such as spin lock detection $[5,6]$ or the use of multiple echoes [4].

For our experimental conditions, a simpler expression may be used to describe the possible enhancement obtained with remote detection. For a first approximation signal-to-noise ratio comparison of remote and direct detection, we assume that the same number of spins, therefore same value of $m_{0}$ is detected using either method. We encode and detect in the same static magnetic field ( $\omega_{0}$ remains constant), both coils were at approximately the same temperature, and the $T_{2}{ }^{*}$ (thus detection bandwidth $\Delta f$ ) of the signal is comparable in both coils. Therefore, the sensitivity of our experiment is only improved through optimization of coil $Q$ and $V_{c}$. The $B_{1}$ field amplitude, and thus $t_{90}(\pi / 2$ pulse length) is directly related to these parameters [23], as expressed in eqn. 2.

$$
S \propto m_{0} \cdot \sqrt{Q / V_{c}} \propto m_{0} \cdot B_{1} \propto m_{0} \cdot 1 / t_{90}
$$

Consequently, knowledge of the $t_{90}$ of both coils-for the same applied powerallows us a rough calculation of the expected signal-to-noise ratio improvement, in agreement with the principle of reciprocity [22]. 


\section{RESULTS AND DISCUSSION}

Two remote images, obtained by Fourier-Transform NMR utilizing pulsed field gradients, are presented. One phantom sample was a Kel-F cylinder with the letters CAL of $0.7 \mathrm{~mm}$ thickness carved through the cylinder axis, so that the flowing xenon gas occupied only the void-space in the shape of the letters. The effective fractional void volume accessible to the xenon gas was $\sim 0.25$ resulting in a filling factor of 0.125 . The second sample was a $14 \mathrm{~mm}$ diameter, $10 \mathrm{~mm}$ long glass cylinder with $1.4 \mathrm{~mm}$ diameter cylindrical pores all the way through the cylinder axis, which is also the direction of gas flow. This porous sample resembles a $2 \mathrm{D}$ honeycomb structure with a fractional void volume of $\sim 0.45$ and filling factor 0.225 . In both cases the projection of the gas on to the plane perpendicular to the flow direction was imaged. While the first image demonstrates the ability to accurately reconstruct fine structures with superior quality by the remote detection technique, the second image illustrates the feasibility of applying this technique to complex structures, potentially to real porous materials.

Before images were acquired with the pulse sequence shown in fig. $2 a$ and $2 b$, the travel time required for the encoded Xe-129 spins to reach the detection location was determined for a given flow path and rate as follows. In the encoding cell a $\pi$-pulse was applied to invert the Xe-129 magnetization. This served as a tag to allow observation of the encoded Xe-129 upon arrival at the detection coil. Following the inversion pulse, we began pulsing repeatedly at the detection coil in order to continually observe the arrival of the inverted 
magnetization from the encoding coil. An example of a travel time curve, a plot of signal from the detection coil versus flow time, is shown in fig. 2c. For short flow times, the encoded Xe-129 had not yet reached the detector, so the detection signal shows maximum intensity. At the flow time corresponding to the travel time, when encoded Xe-129 had fully reached the detector, the detected signal would be expected to have maximum negative intensity. However, due to spreading and mixing with unencoded xenon, the resulting signal generally had only small (negative or positive) amplitudes. Nevertheless, the spectrum with the smallest signal amplitude corresponded to the travel time for which the maximum amount of encoded Xe-129 and minimum amount of unencoded Xe-129 had occupied the detection cell. At flow times longer than the travel time the signal intensity slowly recovered to its maximum. During an imaging experiment, in order to compensate for the signal loss due to the spatial spread of encoded signal during the traveling period, the information carrying longitudinal magnetization of the spins is collected by pulsing and acquiring several times for each encoded point in k-space. Each of these acquisitions corresponds to the detection of an encoded spin volume arriving at a different time. In the following we will refer to each of such detected volumes of spins a batch of spins. The appropriate number of batches and timing was determined by means of the travel time curve.

The pulse sequence used to acquire the 2D pulsed FT remote images (fig. 2) consisted of an excitation pulse, an evolution period during which two orthogonal gradients were applied perpendicular to the direction of flow, and a 
storage pulse. The gradient duration was $150 \mu \mathrm{s}$ and the amplitude was arrayed in 128 steps with an increment of $1.56 \mathrm{G} / \mathrm{cm}$. After the spatial information was encoded in the Xe-129 gas, it flowed to the detection cell. At the detection cell a $\pi / 2$ pulse was applied and the resulting free induction decay (FID) was acquired with an acquisition time of $12.8 \mathrm{~ms}$. Each FID collected at the detection cell provided one point of the indirect dimension $k$-space signal. This collection of signal in the detection coil was repeated 10 times for each encoding point so as to collect the encoded Xe-129 which spread spatially during travel. The minimum pulse delay between each acquisition depended on the flow rate with which the Xe-129 traveled through the coils and was replaced by "fresh" gas. With our experimental setup, a rapid pulsing with a repetition delay as short as $100 \mathrm{~ms}$ could be realized. Consequently, a $128 \times 1282 \mathrm{D}$ remote image obtained by a 3D sequence required less than 2 hours total experimental time. Only one average was taken in order to save time, though with two averages and phase cycling the complete complex image could have been acquired. In this case, there was no advantage to doing this since the image did not overlap with the axes and thus the lack of frequency discrimination did not lead to any ambiguity in image features.

The remote image presented in fig. 3a was the result of a composite of 10 batches. The first batch was acquired in the detection cell $80 \mathrm{~ms}$ after it was encoded in the encoding cell. The subsequent batches were acquired $13 \mathrm{~ms}$ apart, which was ample time for the previous Xe-129 batch to exit the cell volume (at a flow rate of $16 \mathrm{ml} / \mathrm{s}$ ). If the flow from the encoding to the detection coil 
would follow a perfect time-invariant flow pattern, each batch of Xe-129 taken out of the pore volume of "CAL" should have basically the same features, so that adding them together would simply lead to a signal-to-noise gain by the square root of the number of batches. However, the geometry of the cells did not facilitate regular flow. Since we used solenoid coils, the gas flow path necessarily went around corners. One consequence of this disturbed flow pattern was that encoded gas near the top of the cell, which was closer to the outlet, reached the detector sooner than the gas at the bottom of the cell. Also, the gas possibly encountered the bottom corner of the cell upon exit, creating a turbulence that may have further delayed its arrival at the detection cell. This flow pattern was observed by processing each batch independently. The remote image in fig. $3 b$ was comprised of a sum of earlier batches containing only the top of the "CAL" image while subsequent batches filled in the rest of the image slice by slice. Figure $3 c$ shows the sum of intermediate batches. The sum image in fig. $3 \mathrm{~d}$ of later batches visualizes the bottom of the sample. The complete image shown in fig. $3 a$ is the sum over all batches.

The remotely-detected 2D cross-sectional images of the "honeycomb" phantom (fig. 4a) were measured with the same experimental conditions as for the "CAL" phantom. This Xe-129 void space image gives an impression of the fine structure of the honeycomb phantom (fig. 4b) which emulated a porous material. The porous glass phantom was placed loosely in the encoding cell, which allowed some gas to flow around it. The higher intensity at the edges of the image makes this evident. By close inspection one can identify regions in the 
middle section and edges of the phantom where the pores and cracks match with the real structure verified in the photograph. The close-up image presented in fig. 4c supports this observation.

For comparison, a 2D image of each phantom was directly detected in the encoding cell using the same experimental parameters. But instead of storing the signal after each evolution period, it was detected directly by the encoding coil. The major difference was the sensitivity of the detector. The direct images, shown in fig. 5a, b were acquired by means of a large encoding coil, while the remote images (fig. $5 \mathrm{c}, \mathrm{d}$ ) - encoded with the same coil and thus same poor filling factor-were detected with a smaller detection coil.

An order of magnitude calculation of sensitivity enhancement when employing remote detection can be made using eqn. 2 . The enhancement obtained in our remote MRI experiment is mainly due to the difference in quality factors and coil volumes which are reflected in the $t_{90}$ for each coil. For the honeycomb images presented here, the ratio of $t_{90}$ for the encoding coil to $t_{90}$ for the detection coil is $\sim 30$. The actual enhancement obtained using remote detection was about 4 times. In the case of the "CAL" phantom, the ratio of $t_{90}$ for the encoding coil and the detection coil was $\sim 2$. The actual enhancement was approximately 1.7 times. However this is merely an order of magnitude calculation and other factors complicate a quantitative comparison between direct and remote images. For the maximum enhancement to be achieved, all of the encoded signal must be detected at the detection coil. Though information obtained from the travel time curve was used to ensure quantitative acquisition of 
all encoded Xe-129, there was no sharp cut-off at which the gas fully retained its initial polarization. Some portion of the encoded gas was not accounted for in the signal accumulation of 10 batches. This had two different effects on the remote images, namely a decrease in the actual compared to the anticipated signal-tonoise enhancement and in some cases, an enhancement in image resolution.

A compromise between the signal-to-noise and resolution enhancement of the remote image with respect to the direct image may be observed in figure 5 . In the case of the honeycomb image, less signal is detected in the remote image than in the direct image. Whereas the direct image corresponds to a projection along the cylinder axis originating from the whole excitation volume, the remote image corresponds to a projection along a shorter axis length. The latter arises because only those spins that arrive at the detection coil within the predetermined number of batches get collected. These spins originate from a smaller and more homogeneous encoding cell volume. This leads to a superior image resolution of the remote image due to longer living signal, though with fewer Xe-129 spins detected. Therefore, the signal-to-noise enhancement is much less than expected.

However, the comparison between the direct and remote images of the CAL structure (fig. 5b,d) illustrate a different situation. In this case, the "CAL" remote image has a comparable resolution to the analogous direct image but a much higher signal-to-noise ratio. This indicates that a large portion of the encoded Xe-129 atoms were collected at the detection coil under superior detection conditions. 
In addition to these factors, indirect dimension signal inherently suffers from $t_{1}$ noise which results from fluctuations in the signal from point to point, that in the direct dimension would only be manifest as a slight shift in signal intensity. These effects will be discussed more thoroughly in future work.

\section{EXPERIMENTAL}

The images presented were acquired in a $7 \mathrm{~T}$ superconducting magnet equipped with a set of actively shielded micro-imaging gradient coils (Varian) and a 4-cm diameter home-built narrow-bore remote detection probe. The two-coil remote imaging probe consists of an encoding solenoid coil, large enough to surround the sample of interest, and a smaller detection solenoid coil with a higher quality factor. The proximity of the two coils caused a noise problem originating from the decoupler channel of the spectrometer (Varian, Unity Inova), which is used to pulse on the encoding coil. The detection coil, inductively coupled to the encoding coil, picked up some of this noise since both coils were necessarily tuned to the same frequency $(83 \mathrm{MHz})$ and were only separated by a length of $3 \mathrm{~cm}$. This was minimized by orienting the coils as perpendicular to one another as geometric constraints would allow and soldering copper shielding to ground around each coil.

The probe was placed inside the imaging magnet such that the most homogeneous region was positioned between the coils but closer to the encoding coil. While the homogeneity of the encoding region is directly related to 
the image quality, the homogeneity of the detection region is not as crucial for the image resolution. This is because the overall integral of the direct dimension signal, not the characteristics of the signal such as line width, shape, and amplitude are relevant for the point-by-point detected remote signal modulation along the indirect dimension. Therefore, the line broadening due to inhomogeneity in the detection region does not necessarily lead to signal loss along the indirect dimension and can even be mitigated by effective narrowing of the detection bandwidth. The gradient coils were centered around the encoding coil since the gradients were only used for encoding.

Hyperpolarized Xe-129 in natural abundance was prepared from a Xe: $\mathrm{N}_{2}: \mathrm{He}(1: 2: 97)$ gas mixture (Spectra Gases) using a commercial polarizer (Amersham Health). The experiments were performed in continuous flow mode with the higher pressure $(\sim 7 \mathrm{~atm})$ in the pumping cell providing the force to drive the gas from the polarizer to the first coil for encoding and subsequently to the second for sensitive detection. The outlet of the detection cell was connected to a long thin plastic tube with a narrow opening at the end. This restriction helped the stabilization of the flow rate and prevented oxygen from diffusing back into the system which would have led to faster relaxation of the Xe-129 polarization. It was essential to maintain a stable flow rate, since the actual travel time of $\mathrm{Xe}$ 129 between the encoding coil and the detection coil is accurately matched with the timing of the pulse sequence. As a result, an irregular flow rate, thus travel time, would lead to a fluctuation of the signal amplitudes along the indirect dimension. 


\section{CONCLUSION}

The proper application of the NMR remote detection technique including the controlled and quantitative transport of encoded spins from the imaging to the detection region leads to higher sensitivity and maximum image resolution. Homogeneity in $B_{0}$ and $B_{1}$ field in the encoding region and a sensitive detector are often required for the successful implementation of remote detection although inhomogeneous broadening can be compensated for by the use of nutationecho/ex-situ methodology [24, 25].

Our results demonstrate how a certain flow pattern can influence the resulting image features. A non-uniform flow pattern and the mismatch in sample volumes of the encoding and detection cell led to a partitioning of the encoded gas volume, so that the detection step had to be repeated over a certain period to collect the information in several batches. On the other hand, such information is useful to study flow properties. The experimental time can be vastly reduced by employing stop-and-go flow control systems and utilizing pumping or injection devices. Also, the use of upstanding coils, and thus a straight flow path will provide a more efficient transfer of gas between coils, resulting in a smaller volume of gas that contains encoded xenon. With a smaller volume of gas to be detected, options such as the adoption of microcoils [26-28] become viable for detection. With further sensitivity gain afforded by these improvements higher gradients can be utilized to image materials. Ultimately, this technique can be applied to resolve the fine structure of various living and inorganic porous 
materials that are not accessible by conventional NMR imaging methods within a reasonable experimental time.

\section{ACKNOWLEDGEMENTS}

We thank Josef Granwehr for valuable discussions and kindly reviewing the manuscript. Hardware advice regarding probe construction from Rachel Martin, and the experimental support for setting up the remote detection technique of Adam Moule and Kimberly Pierce are gratefully acknowledged. This work was supported by the Director, Office of Science, Office of Basic Energy Sciences, Materials Sciences of the U.S. Department of Energy under Contract No. DEAC03-76SF00098. Song-I Han gratefully acknowledges the Alexander von Humboldt Foundation for support through a postdoctoral fellowship. Technical support of Eric Granlund for sample construction and Jim Breen for the glassware fabrication is acknowledged.

\section{REFERENCES}

[1] A. J. Moule, M. M. Spence, S-I. Han, J. A. Seeley, K. L. Pierce, S. Saxena, Pines, A., Amplification of Xenon NMR and MRI by Remote Detection. Proc. Nat. Acad. Sci 100 (2003) 9122.

[2] R. McDermott, et al, Liquid-state NMR and scalar couplings in microtesla magnetic fields. Science 295 (2002) 2247.

[3] D. Raftery, H. Long, D. Shykind, P. J. Grandinetti, A. Pines, Multiple-pulse nuclear- 
magnetic-resonance of optically pumped Xenon in a low magnetic field. Phys. Rev. A. 50 (1994) 567.

[4] D. P. Weitekamp, Time-domain multiple-quantum NMR. Adv. Magn. Reson. 11 (1983) 111.

[5] K. H. Lim, T. Nguyen, T. Mazur, D. E. Wemmer, A. Pines, Sensitivity enhancement in multiple-quantum NMR experiments with CPMG detection. J. Magn. Reson. 157 (2002) 160.

[6] O. N. Antzutkin, R. Tycko, High-order multiple quantum excitation in 13-C nuclear magnetic resonance spectroscopy of organic solids. J. Chem. Phys. 110 (1999) 2749.

[7] B. M. Goodson, Nuclear magnetic resonance of laser-polarized noble gases in molecules, materials, and organisms. J. Magn. Reson. 155 (2002) 157.

[8] T. Pietrass, H. C. Gaede, Optically polarized Xe-129 in NMR-spectroscopy. Adv. Mater. 7 (10) (1995) 826.

[9] T. Ito, J. Fraissard, Xe-129 NMR-study of Xenon adsorbed on Y zeolites. J. Chem. Phys. 76 (11) (1982) 5225.

[10] R. F. Tilton, I. D. Kuntz, Nuclear magnetic-resonance studies of Xe-129 with myoglobin and hemoglobin. Biochemistry 21 (26) (1982) 6850.

[11] J. P. Mugler, et al., MR imaging and spectroscopy using hyperpolarized Xe-129 gas: Preliminary human results. Magn. Reson. Med. 37 (6) (1997) 809.

[12] M. S. Albert, et al., Biological magnetic-resonance-imaging using laser polarized Xe-129. Nature 370 (6486) (1994) 199.

[13] C. J. Jameson, A. C. de Dios, Xe nuclear magnetic resonance line shapes in nanochannels. J. Chem. Phys. 116 (9) (2002) 3805.

[14] A. Kastler, Quelques suggestions concernant la production optique et la detection optique dune inegalite de population des niveaux de quantification spatiale des 
atomes- application a lexperience de stern et getlach et a la resonance magnetique. J. Phys. Radium 11 (6) (1950) 255.

[15] W. Happer, et al., Polarization of the nuclear spins of noble-gas atoms by spin exchange with optically pumped alkali-metal atoms. Phys Rev. A 29 (6) (1984) 3092.

[16] S. Appelt, et al., Theory of spin-exchange optical pumping of He-3 and Xe-129. Phys Rev A 58 (2) (1998) 1412.

[17] M. Springuel-Huet, J. Bonardet, A. Gedeon, J. Fraissard, Xe-129 NMR overview of xenon physisorbed in porous solids. Magnetic Reson. In Chemistry 37 (1999) S1.

[18] S. M. Rubin, et al., Detection of a conformational change in maltose binding protein by Xe-129 NMR spectroscopy. J. Amer. Chem. Soc. 123 (35) (2001) 8616.

[19] M. M. Spence, et al., Functionalized xenon as a biosensor. Proc. Nat. Acad. Sci. 98 (19) (2001) 10654,.

[20] E. Locci, J. Reisse, K. Bartik, The potential of the xenon "Spin-Spy" methodology for the study of configurational equilibria in solution. Chem. Phys. Chem. 4(3) (2003) 305.

[21] Callaghan, P.T., "Principles of NMR Microscopy”, Oxford: Clarendon Press, 1991.

[22] D. I. Hoult, R. E. Richards, The Signal-to-Noise Ratio of the Nuclear Magnetic Resonance Experiment. J. Magn. Reson. 24 (1976) 71.

[23] Fukushima, E., Roeder, S.B.W., "Experimental Pulse NMR: A Nuts and Bolts Approach," Massachusetts: Addison-Wesley Publishing Company, 1981.

[24] A. Sharfenecker, I. Ardelean, R. Kimmich, Diffusion measurements with the aid of nutation spin echoes appearing after two inhomogeneous radiofrequency pulses in inhomogeneous magnetic fields. J. Magn. Reson. 148 (2001) 363.

[25] C. A. Meriles, D. Sakellariou, H. Heise, A. J. Moule, A. Pines, Approach to highresolution ex-situ NMR spectroscopy. Science 293 (5527) (2001) 82. 
[26] E. W. Mc Farland, A. Morata, 3-dimensional NMR microscopy-improving SNR with temperature and microcoils. Magn. Reson. Imag. 10 (2) (1992) 279.

[27] A. G. Webb, Radiofrequency microcoils in magnetic resonance. Prog. Nucl. Magn Reson. Spectrosc. 31 (1997) 1.

[28] D. L. Olson, T. L. Peck, A. G. Webb, R. L. Magin, J. V. Sweedler, High-resolution microcoil H-1-NMR for mass-limited, nanoliter-volume samples. Science $\mathbf{2 7 0}$ (1995) 1967.

\section{FIGURE LEGENDS}

Figure 1. Remote detection schematic. The dilute sensor inside a sample matrix is encoded and stored by a large coil with a poor filling factor and physically transported to a different location for optimized detection. The stored signal can be read out more sensitively with a smaller RF-coil, or by alternative detection techniques such as SQUID and optical detection. The data presented in this article was acquired with a small, sensitive Faraday coil.

Figure 2. Remote imaging pulse sequence and travel time curve. a) The remote imaging encoding sequence consists of an excitation pulse, an evolution period during which two orthogonal gradients are applied perpendicular to the direction of flow, and a storage pulse. The gradients are independently arrayed 128 times for $150 \mu \mathrm{s}$. with an amplitude increment of $1.56 \mathrm{G} / \mathrm{cm}$. After the spatial information is encoded in the Xe-129 gas, it flows to the detection cell. b) At the detection cell a $\pi / 2$ pulse was applied and the resulting free induction decay (FID) was acquired. Each FID collected at the detection cell provided one point 
of the indirect dimension $k$-space signal. This collection of signal in the detection coil was repeated 10 times for each encoding point so as to collect the encoded Xe-129 which spread during travel. c) The travel time data shown was acquired by inverting the magnetization in the encoding coil and then arraying the time the gas was allowed to flow towards the detection coil where it was detected. As the inverted signal reached the detector the signal intensity was most depleted. The flow time corresponding to the minimum intensity was the time at which the maximum amount of encoded signal and the minimum amount of unencoded signal was present at the detection coil. The width of the spread of encoded gas was determined by the amount that the encoded gas mixed with unencoded gas. During the detection period of a remote imaging experiment Xe-129 was repetitively collected as long as encoded signal was present in the detection coil as is illustrated (dotted lines).

Figure 3. Xe-129 remotely-detected void-space image of the "CAL" phantom. a) The phantom is a $13 \mathrm{~mm}$ diameter cylinder of Kel- $\mathrm{F}$ with the letters "CAL" carved through with feature thickness of $0.7 \mathrm{~mm}$ and a height of $10 \mathrm{~mm}$. The complete remote image of the "CAL" phantom is comprised of 10 batches of gas. b) The Xe-129 encoded at the top of the phantom had the shortest distance to travel to the detector and was thus detected in the first few batches. c) The next few batches to reach the detector corresponded to the Xe-129 encoded in the center of the phantom. d) Finally the bottom features of the phantom were visualized after waiting the longest flow time to collect the encoded Xe-129. 
Figure 4. Xe-129 remotely-detected void-space image of the "Honeycomb" phantom. Different photographs have been used to illustrate different aspects of the phantom. The photograph in figure 4a more accurately depicts the shapes of the pores but since it was taken at a slight angle with respect to the sample, the size of the pores appears larger than they are in reality. Conversely, the photograph in figure $4 \mathrm{~b}$ is slightly blurred and thus displays the pore shapes less accurately, yet their relative placement and size more accurately. a) The remotely-reconstructed image of the "honeycomb" phantom shown is comprised of ten batches of encoded Xe-129. b) The photo of the $14 \mathrm{~mm}$ diameter "honeycomb" phantom with $1.4 \mathrm{~mm}$ pores is shown for comparison. c) A closeup view of selected pores in the center region of the phantom is shown to verify the accuracy of the image.

Figure 5. Comparison of remote and direct void-space images of "CAL" and "honeycomb" phantom. a) The direct image of the "CAL" phantom was acquired by the less sensitive encoding coil. b) The direct image of the "honeycomb" phantom was acquired in the same encoding coil. c) The remotely-detected image of the "CAL" phantom, comprised of 10 batches of encoded Xe-129, was detected more sensitively than the direct image with a smaller coil. d) The "honeycomb" phantom was reconstructed under the same conditions as the image in Fig. 5c, also using 10 batches of gas to ensure the detection of all encoded $\mathrm{Xe}-129$ gas. 
Figure 1

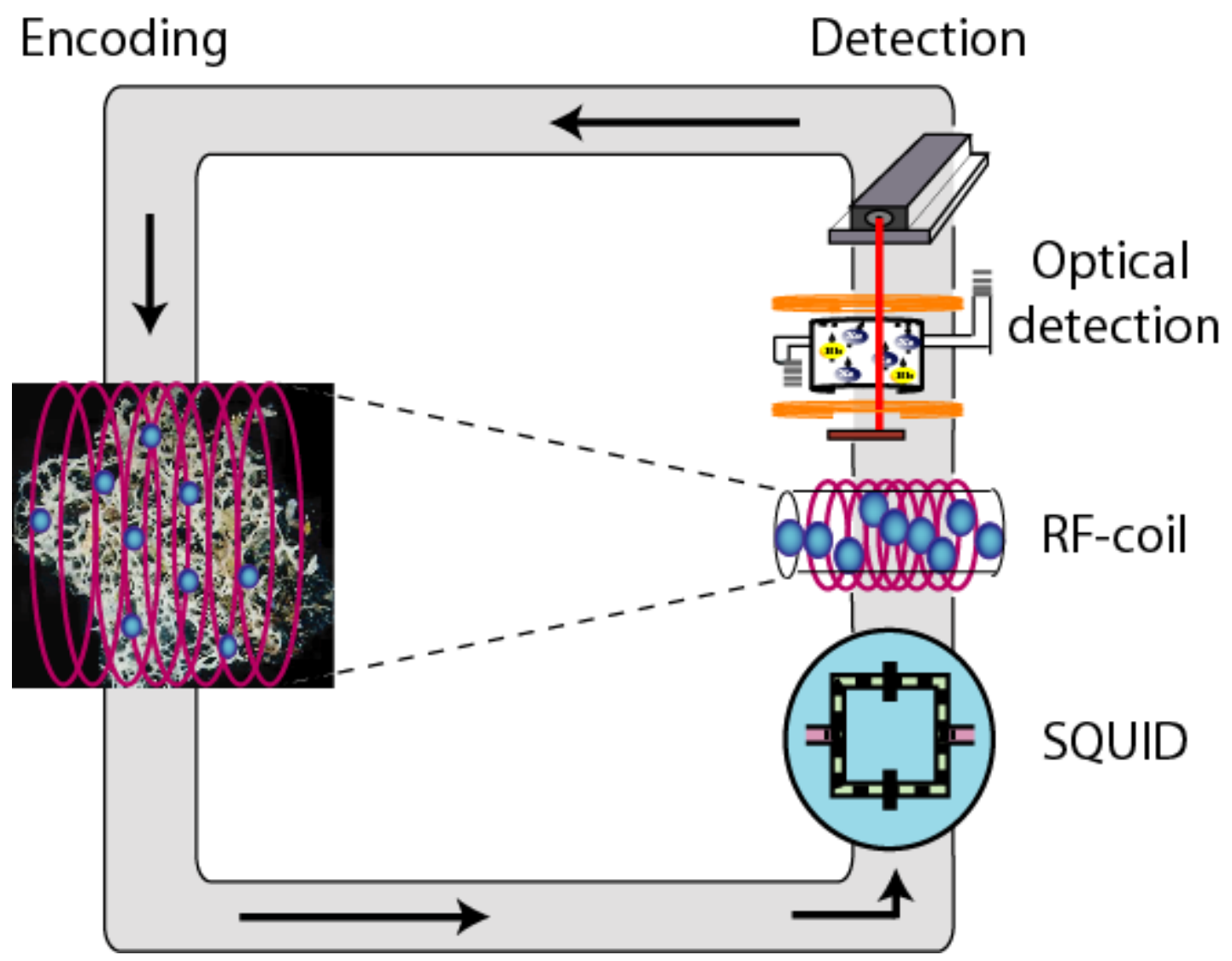


Figure 2

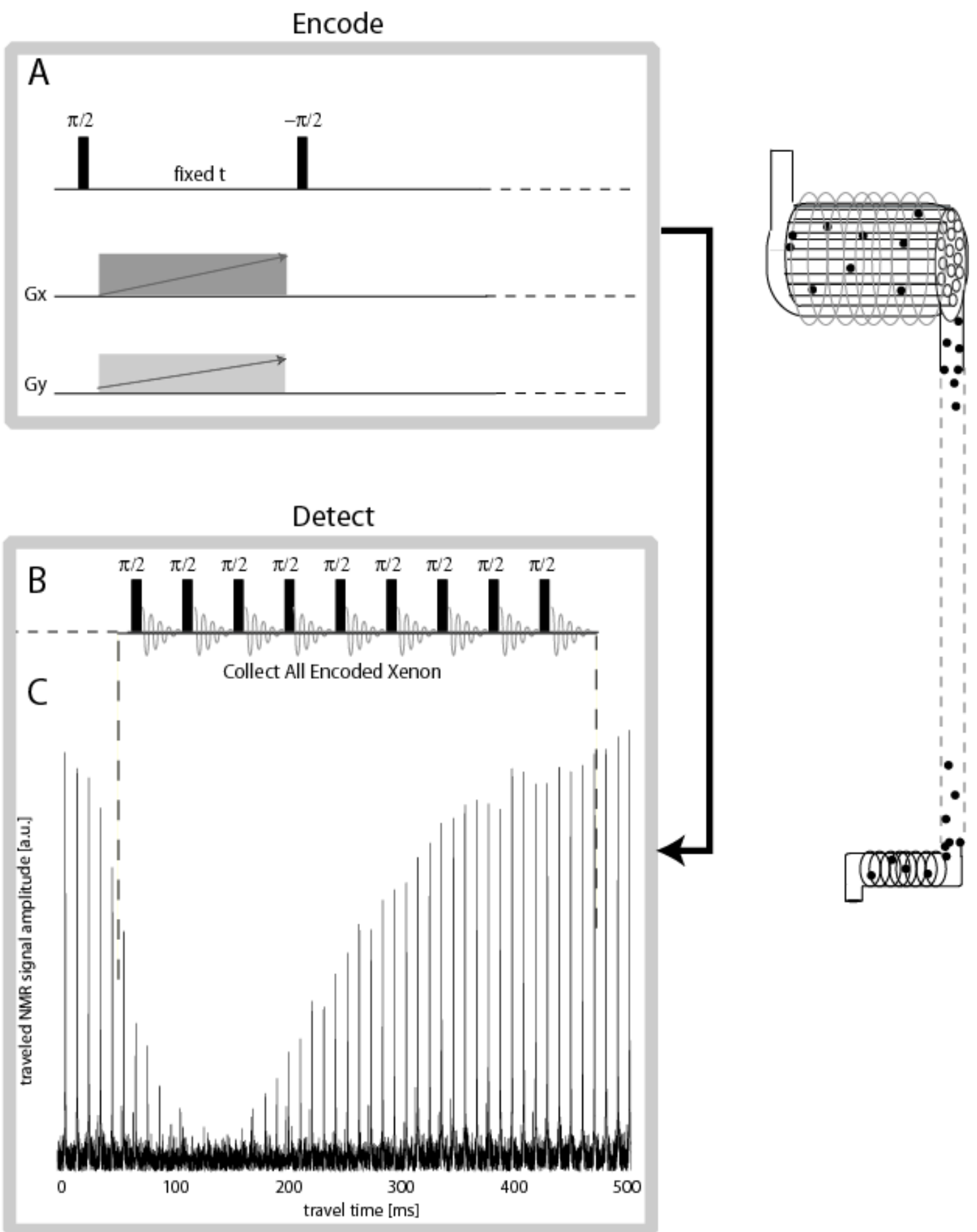


Figure 3

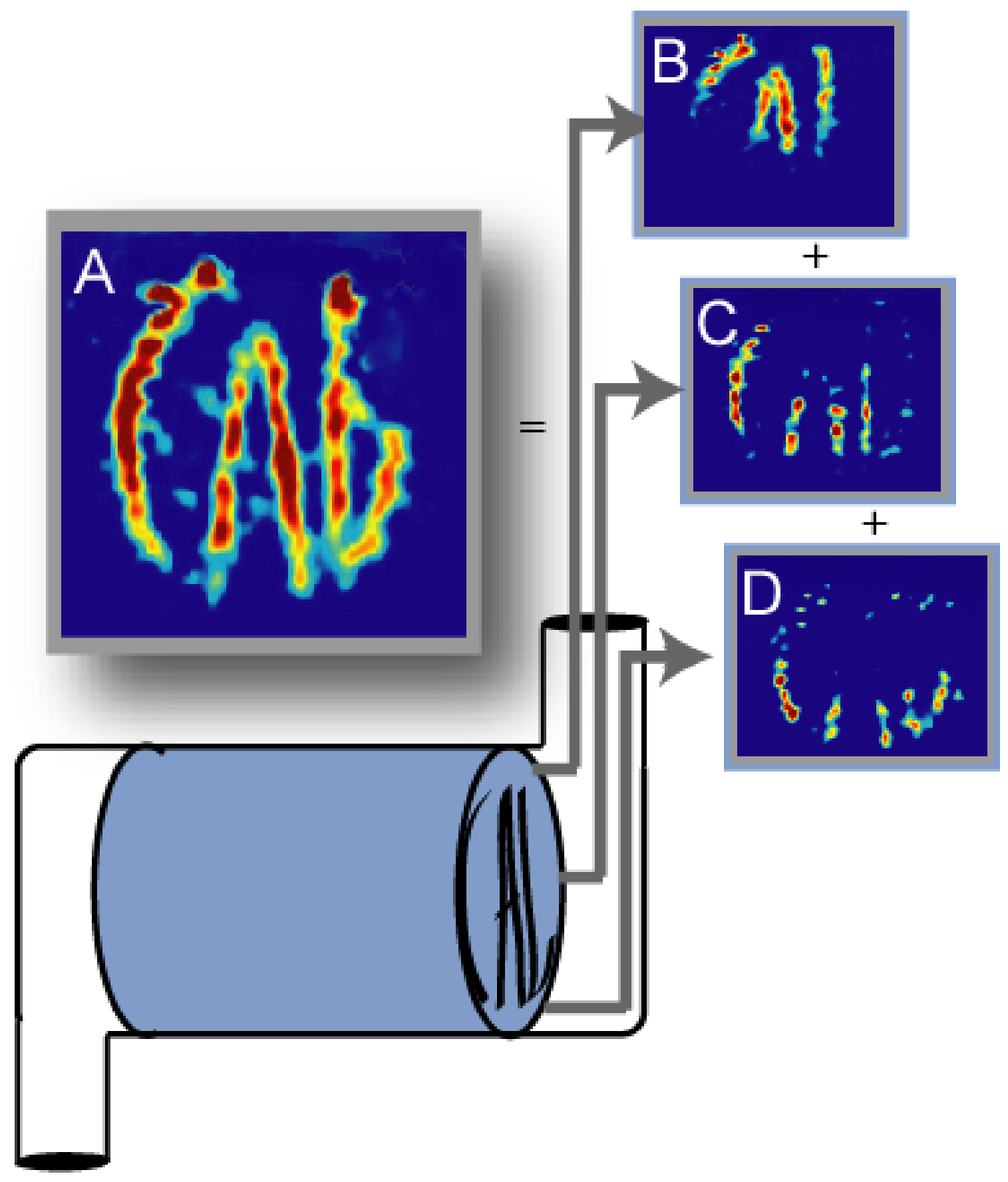


Figure 4
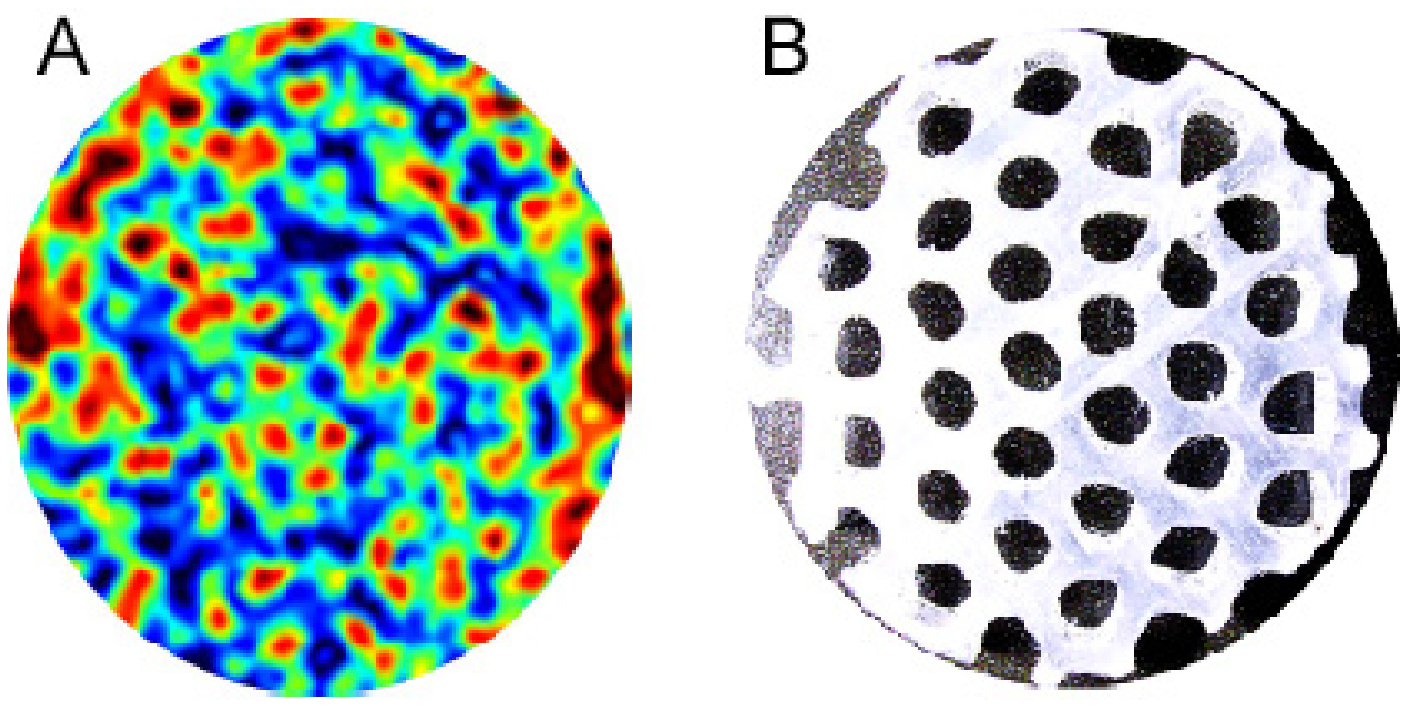

C

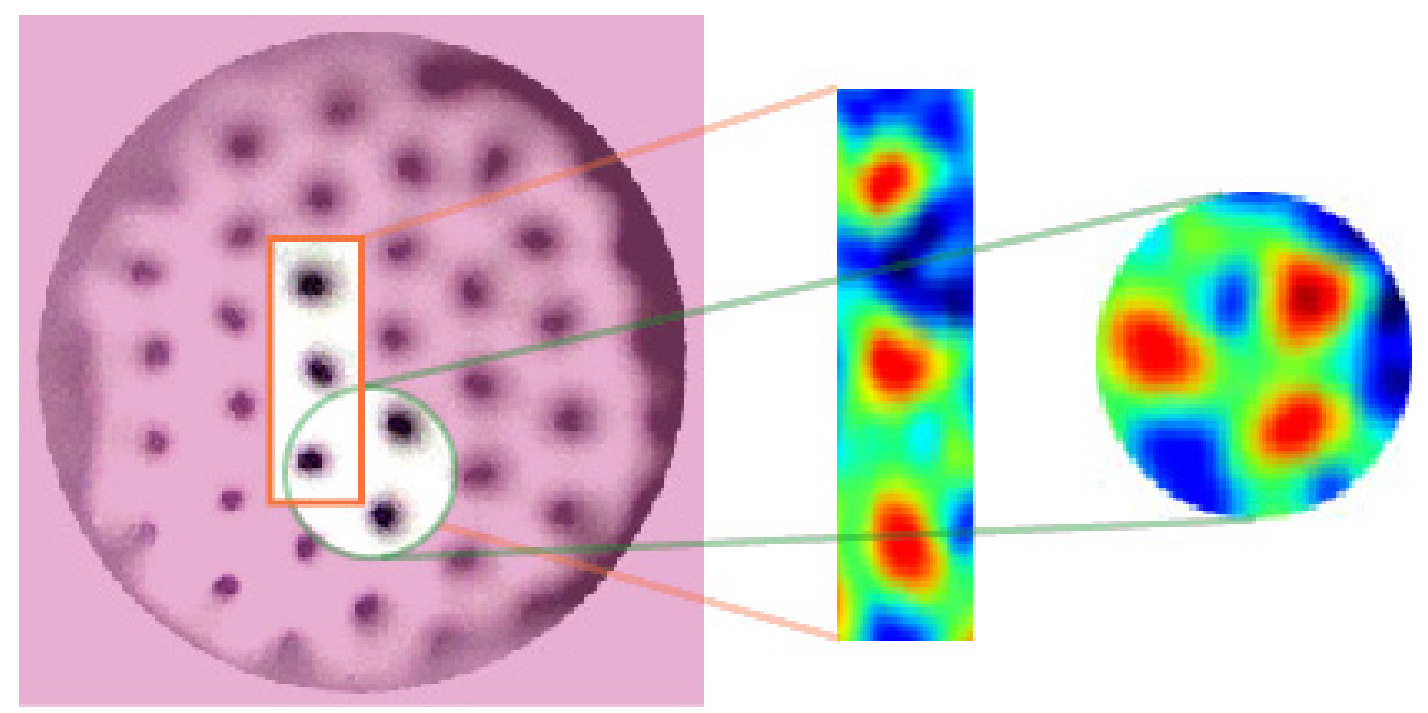


Figure 5
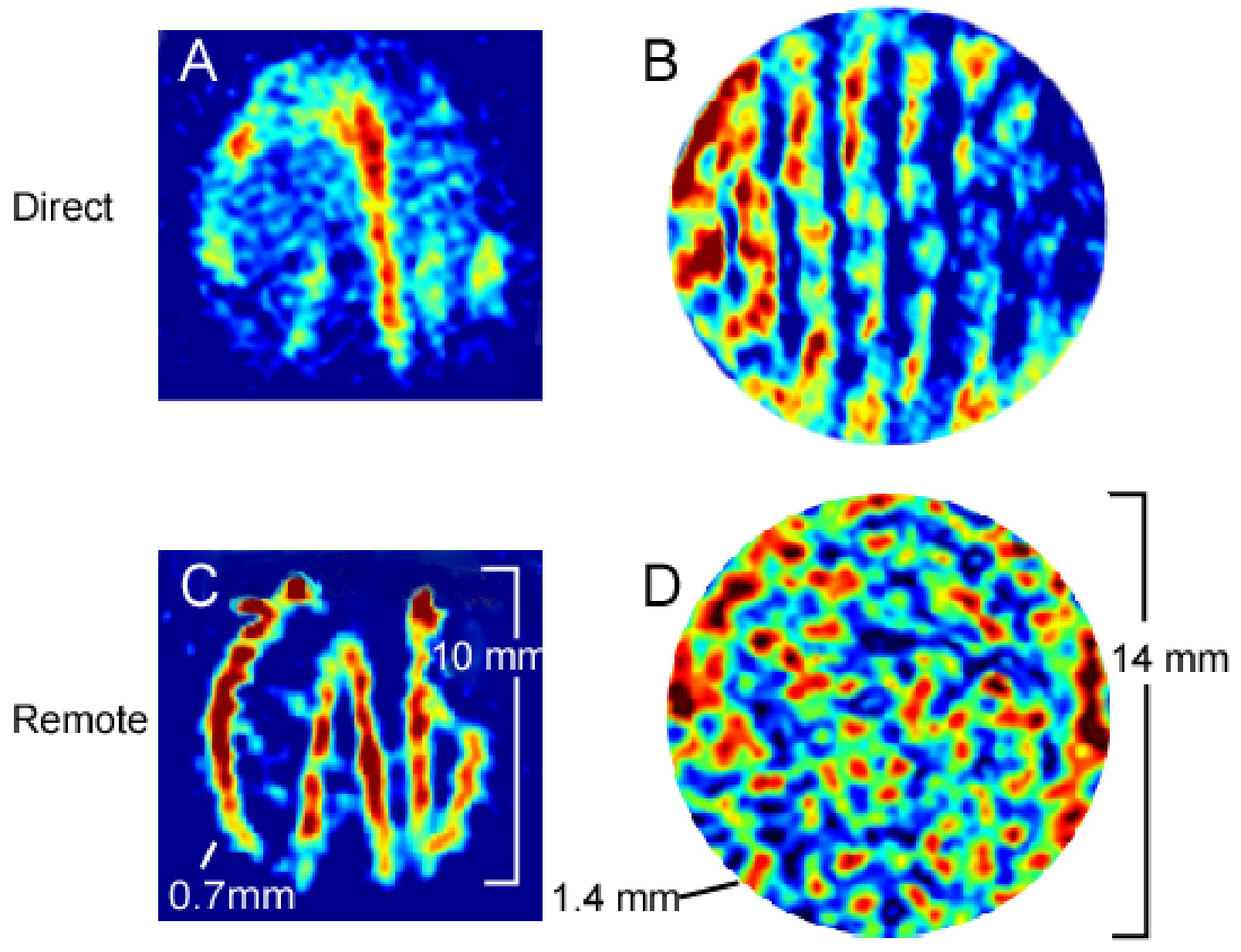
Cover art mock up no.1

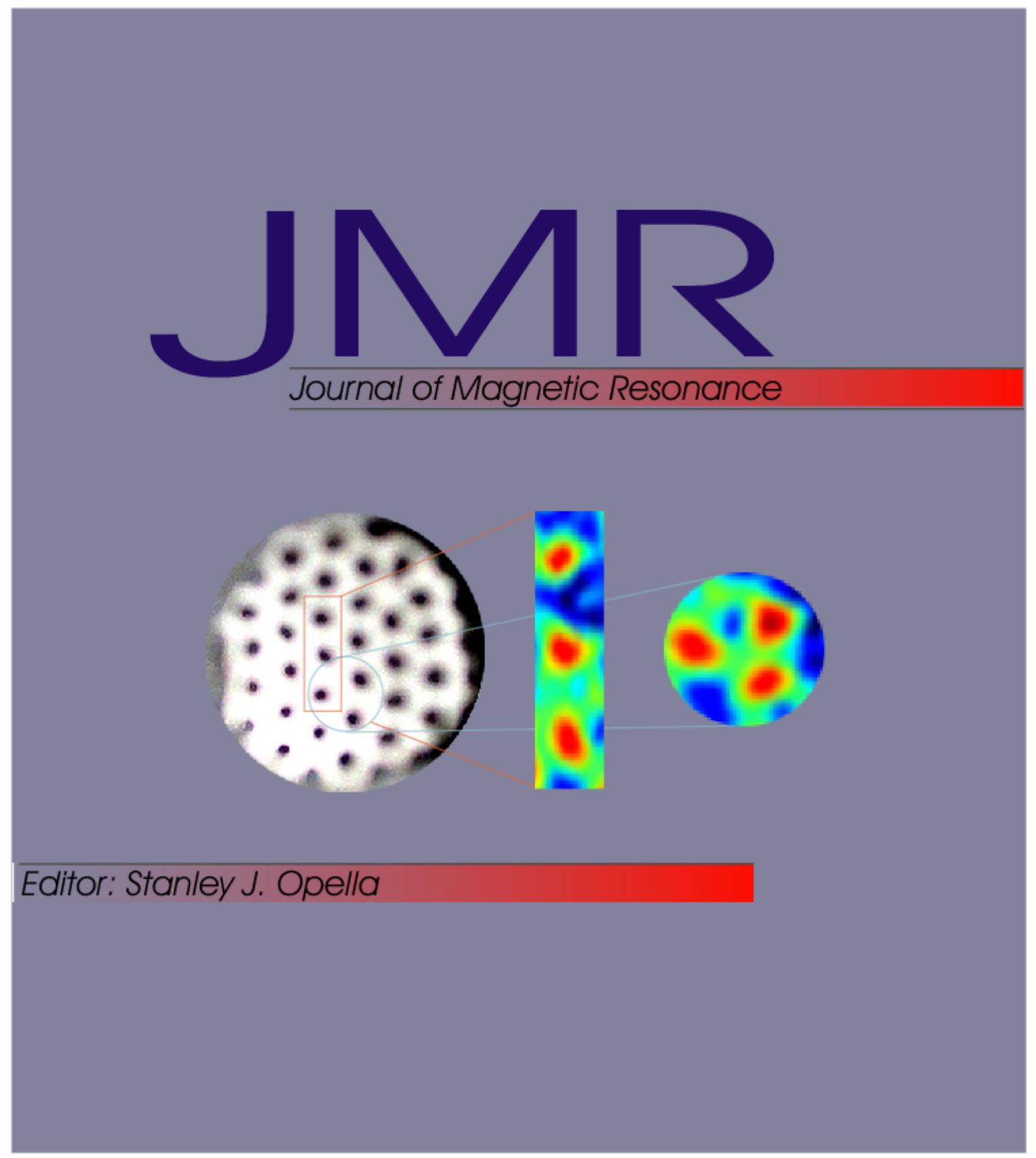


Cover art mock up no.2

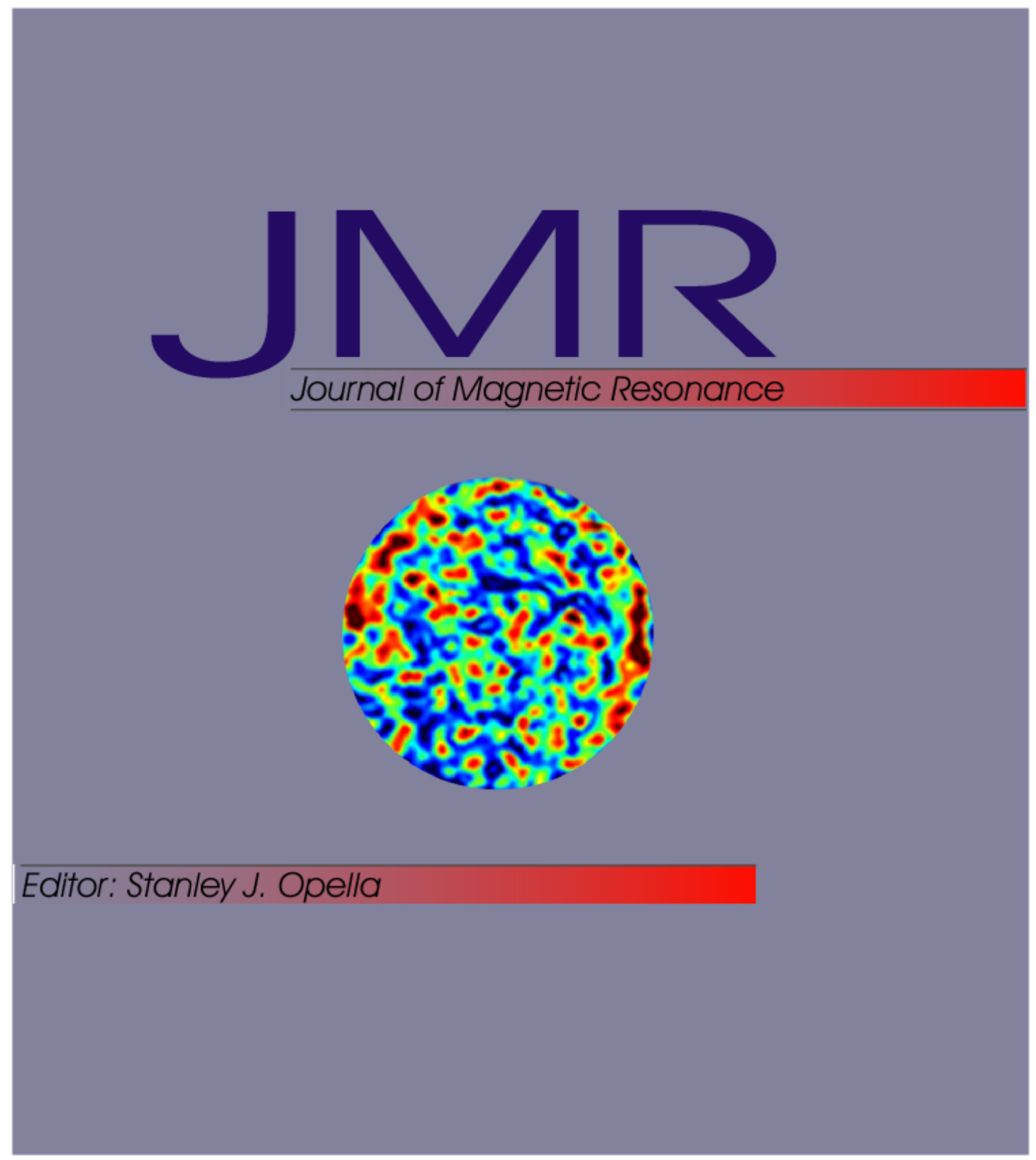


Cover art mock up no.3

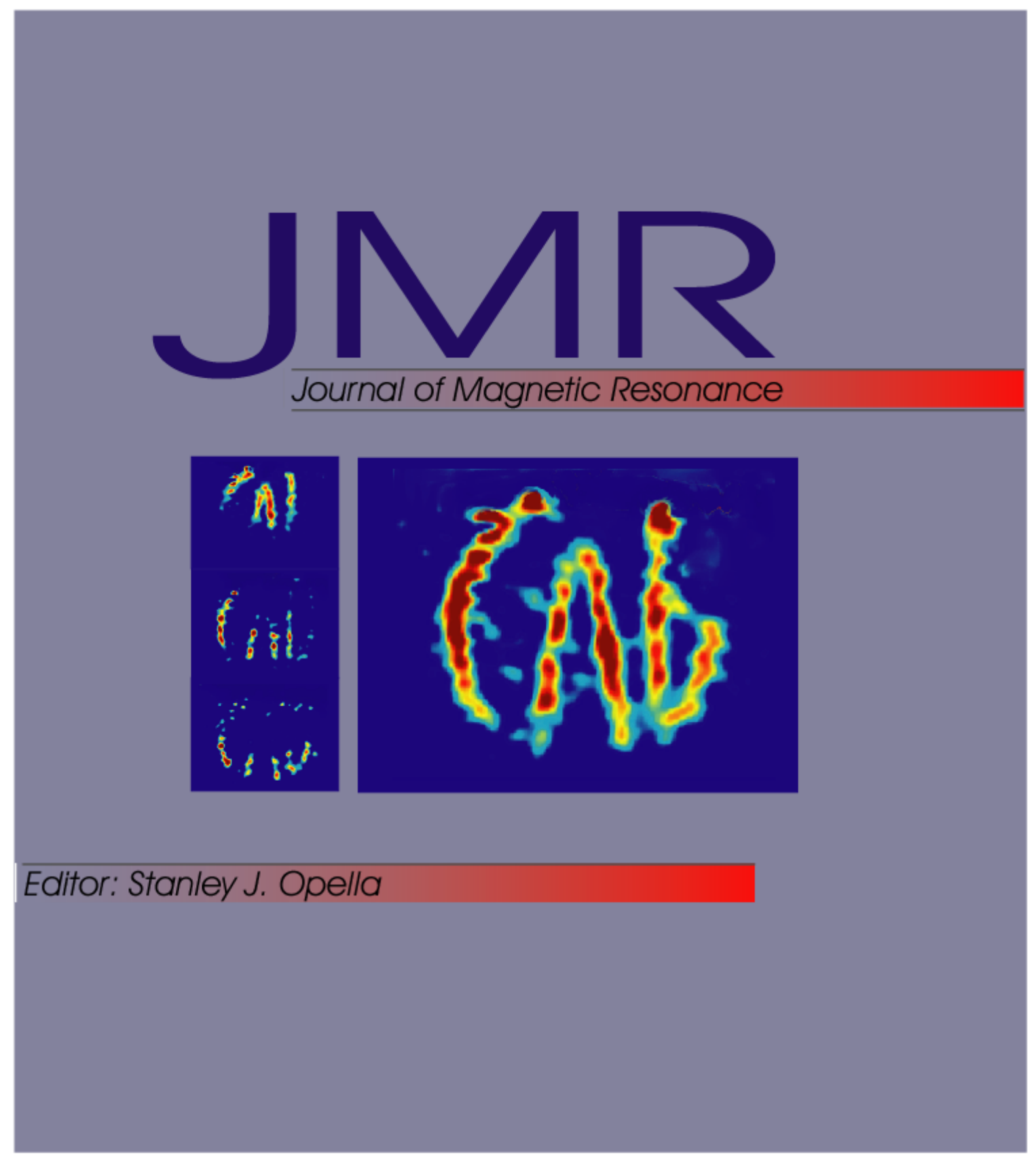

